\title{
Temperature Dependence of the Relaxation Resonance Frequency of Long-Wavelength Vertical-Cavity Lasers
}

\author{
E. Staffan Björlin, Member, IEEE, Jon Geske, Member, IEEE, Manish Mehta, Joachim Piprek, Senior Member, IEEE, \\ and John E. Bowers, Fellow, IEEE
}

\begin{abstract}
The temperature dependence of the differential gain in AlInGaAs 1310-nm vertical-cavity lasers is investigated. The variations in differential gain and in relaxation resonance frequency are shown to depend on the room-temperature offset between the gain peak wavelength and the wavelength of the lasing mode. The tradeoff between high modulation bandwidth and good high-temperature performance for vertical-cavity lasers is analyzed. A cavity mode that is red-shifted about $25 \mathrm{~nm}$ from the gain peak is shown to minimize the variation in modulation bandwidth with temperature, and simultaneously allow for satisfactory high-temperature operation. Experimental results are presented and compared to calculated results with excellent agreement. Because of the change in gain-mode offset with internal temperature, the measured modulation current efficiency changed from about 2 to $4.8 \mathrm{GHz} / \mathrm{mA}^{1 / 2}$ for an increase in drive current from 2 to $10 \mathrm{~mA}$.
\end{abstract}

Index Terms-Optical communication, optical modulation, semiconductor lasers, surface-emitting lasers, vertical-cavity surface-emitting lasers (VCSELs).

V ERTICAL-CAVITY surface-emitting lasers (VCSELs) are of significant interest for application as low-cost sources in optical communication systems. They offer a number of advantages over in-plane devices, such as low power consumption and low manufacturing cost to name a few. For applications in high bit-rate systems, devices with a large modulation bandwidth are required. The modulation bandwidth might in practice be limited by damping or electrical parasitics, but the ultimate bandwidth of a laser is determined by the relaxation resonance frequency of the laser cavity, beyond which the modulation response decreases rapidly. In order to achieve a large relaxation resonance frequency, low threshold current, small mode volume, high efficiency, and a large differential gain are needed. Commonly used methods to maximize the differential gain in high-speed in-plane lasers include employing strained quantum wells (QWs) [1], p-doping the active region [2], or positioning the cavity mode blue-shifted from the gain peak [3].

Long-wavelength VCSELs with relaxation resonance frequencies over $10 \mathrm{GHz}$ at room temperature (RT) have been demonstrated [4]. However, most of the current long-wave-

Manuscript received August 12, 2004; revised November 19, 2004. This work was supported by $\mathrm{E}_{2} 0$ Corporation and by the UC Discovery Grant Program.

The authors are with the Electrical and Computer Engineering Department, University of California, Santa Barbara, Santa Barbara, CA 93106 USA (e-mail: bjorlin@ece.ucsb.edu).

Digital Object Identifier 10.1109/LPT.2005.844560 length VCSEL research is focused on achieving high single-mode power and operation over a wide temperature range [5]-[8]. High temperature operation requires, among other things, a lasing wavelength that is red-shifted with respect to the gain peak, i.e., a negative gain-mode offset. However, the differential gain of a semiconductor laser is higher at short wavelengths and decreases for wavelengths longer than the gain peak. For high-speed operation, it is, therefore, desirable to design the laser with positive or zero gain-mode offset. This well-known fact is routinely utilized to maximize the modulation bandwidth of distributed feedback lasers [3], [9]. For applications such as dense wavelength-division multiplexing, the lasers are temperature controlled so that the emission wavelength does not change during operation and the gain-mode offset, and, therefore, the differential gain, is kept constant. VCSELs, however, typically have larger thermal resistance and also typically target low-cost applications where uncooled operation is desired. Without temperature control, fluctuations in the ambient temperature and self heating caused by changes in drive current lead to variations in gain-mode offset, which consequently cause variations in the VCSEL properties during operation. The alternative source for low-cost applications is the Fabry-Pérot laser, which due to its small mode spacing always lase at the gain peak, i.e., zero gain-mode offset is maintained at all times. The issues discussed here are, therefore, of greater importance for VCSELs compared to most other types of lasers. The differential gain in VCSELs has been analyzed theoretically [10] and experimentally [11], but the temperature dependence of the differential gain and variations with gain-mode offset have not previously been investigated for this class of devices.

Calculated differential gain versus wavelength for an AlInGaAs active region with five compressively strained QWs is shown in Fig. 1. Details about the gain model can be found in [12]. The RT gain peak of the QWs is at $1275 \mathrm{~nm}$. This active region design is consistent with the devices that were used in the experiments presented in this letter. Nine curves are shown, representing active region temperatures from $0{ }^{\circ} \mathrm{C}$ to $160{ }^{\circ} \mathrm{C}$. The gain along the curves is fixed at $550 \mathrm{~cm}^{-1}$, which is the estimated threshold gain of these VCSELs (the slight increase in threshold gain at higher temperatures caused by increased internal loss was determined to not impact the results of these calculations, and is, therefore, neglected in this model). Further details about the device design are reported elsewhere [7], [12]. At low temperatures, the differential gain decreases drastically 


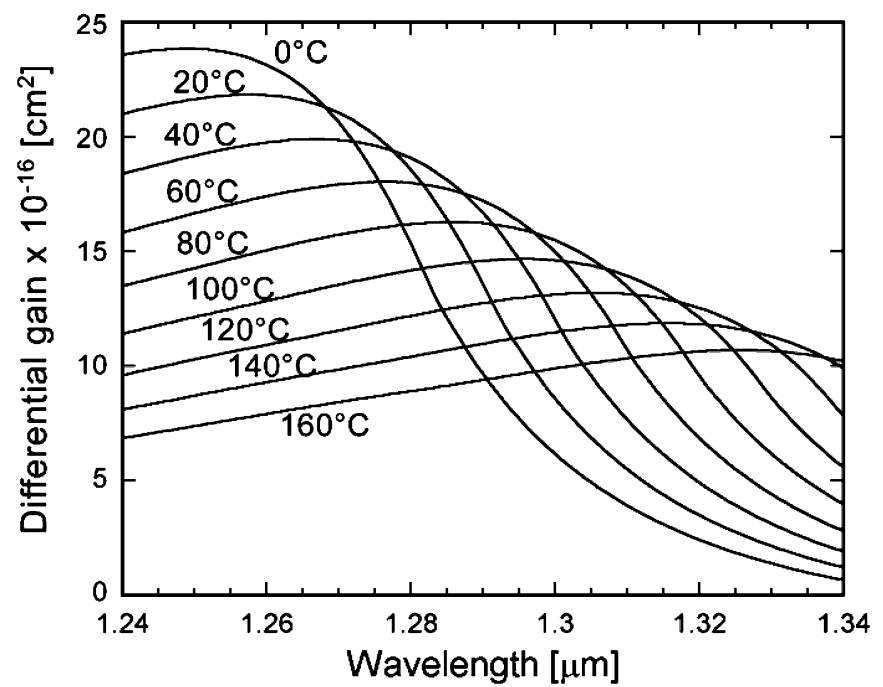

Fig. 1. Calculated differential gain versus wavelength at a constant gain of $550 \mathrm{~cm}^{-1}$, and the RT gain peak at $1275 \mathrm{~nm}$. Curves are shown for active-region temperatures ranging from $0{ }^{\circ} \mathrm{C}$ to $160{ }^{\circ} \mathrm{C}$.

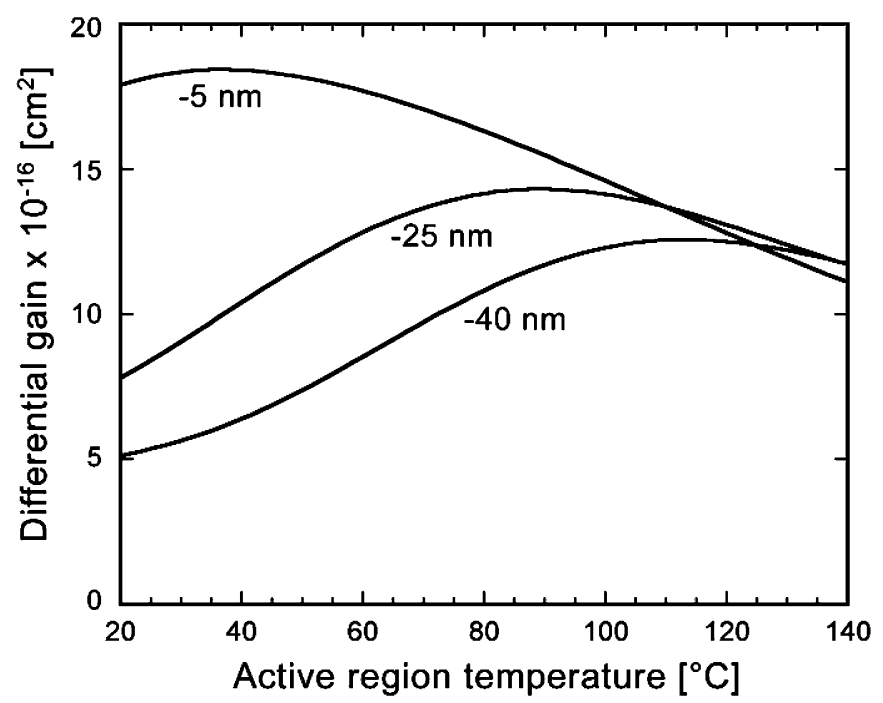

Fig. 2. Calculated differential gain versus temperature for RT gain-mode offsets of $-5,-25$, and $-40 \mathrm{~nm}$.

for wavelengths longer than the gain peak. At higher temperatures, the variation in differential gain is smaller, but the maximum differential gain is also smaller. Over the entire temperature range, the differential gain is highest at a wavelength about $15 \mathrm{~nm}$ shorter than the gain peak.

Fig. 2 shows calculated differential gain versus active-region temperature from $20{ }^{\circ} \mathrm{C}$ to $140{ }^{\circ} \mathrm{C}$, for three different RT gain-mode offsets: $-5,-25$, and $-40 \mathrm{~nm}$. Positive offsets are not considered here, as they are impractical for satisfactory high-temperature operation. The calculations are based on the curves in Fig. 1. A red shift of the cavity mode wavelength of $0.1 \mathrm{~nm} /{ }^{\circ} \mathrm{C}$ was used in the calculations. As expected the highest differential gain can be obtained for the smallest offset at low temperatures. For -5-nm gain-mode offset, the peak value of the differential gain is about $18 \times 10^{-16} \mathrm{~cm}^{2}$ at $35^{\circ} \mathrm{C}$ active region temperature. However, as the temperature increases, the differential gain decreases rapidly. For larger offsets, the differential gain increases with temperature until the cavity

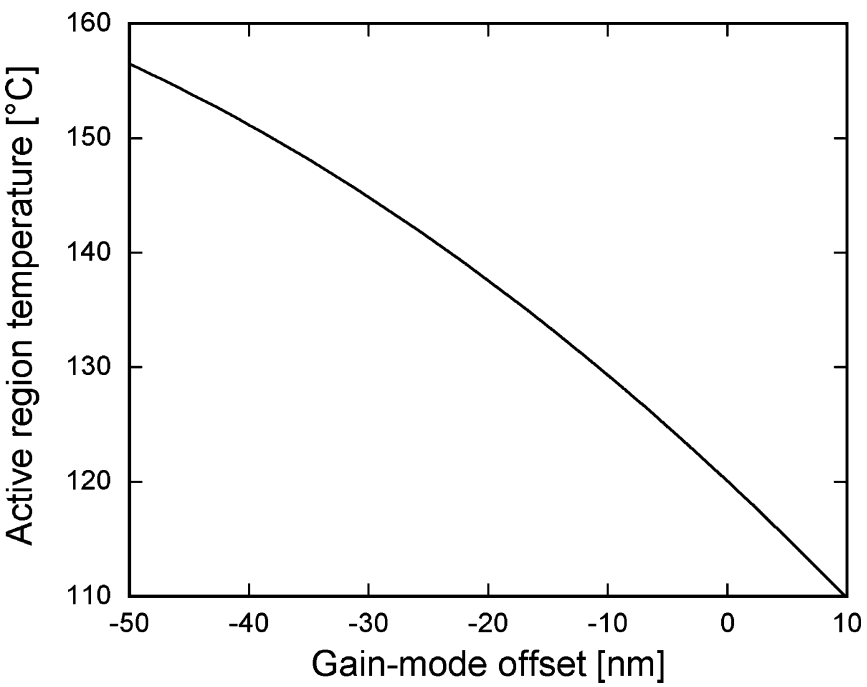

Fig. 3. Maximum lasing temperature versus RT gain-mode offset. A negative offset of about $25 \mathrm{~nm}$ or more is required to achieve lasing at $140^{\circ}$ active region temperature.

mode passes the gain peak. For the QW design analyzed here, -25 -nm offset yields the smallest variation in differential gain, from $10.5 \times 10^{-16} \mathrm{~cm}^{2}$ to a peak value of $14.5 \times 10^{-16} \mathrm{~cm}^{2}$ at about $60^{\circ} \mathrm{C}$ active-region temperature. The VCSELs that were used in this experiment were optimized for high-temperature operation and, therefore, have a large RT gain-mode offset of about $-40 \mathrm{~nm}$. This design is represented by the third curve in Fig. 2. The differential gain of these VCSELs is low at low temperature and increases slowly to a peak value of about $10 \times 10^{-16} \mathrm{~cm}^{2}$ at elevated temperature. It is evident from Figs. 1 and 2 that the highest differential gain can be obtained for small RT gain-mode offsets, provided that the temperature is kept low. To illustrate the design constraints put on the VCSEL by temperature range requirements, gain-mode offset versus maximum lasing temperature (active region temperature) is shown in Fig. 3. The same gain model that was used to generate the data shown in Fig. 1 was used to determine the temperature at which threshold gain can no longer be reached. In order to be able to achieve lasing at $100{ }^{\circ} \mathrm{C}$ ambient temperature, an RT offset of about $-25 \mathrm{~nm}$ or more is required. For an operating bias current of $10 \mathrm{~mA}$, operating voltage of $3 \mathrm{~V}$, and a thermal resistance of $1.3{ }^{\circ} \mathrm{C} / \mathrm{mW}$, this corresponds to about $140{ }^{\circ} \mathrm{C}$ internal temperature.

The calculations are compared to experimental data in Fig. 4. The relaxation resonance frequency $\left(f_{0}\right)$ is plotted versus the square root of the bias current less the threshold current, at $20^{\circ} \mathrm{C}$ ambient temperature. The data, shown as dots, were obtained from relative intensity noise spectra measured on a VCSEL with a $12-\mu \mathrm{m}$ active region diameter and a RT gain-mode offset of $-40 \mathrm{~nm}$. The lines were calculated using the following equation [13]:

$$
f_{0}=\frac{1}{2 \pi}\left[\frac{v_{g} \eta_{i} a}{q V_{m}}\left(I-I_{\mathrm{th}}\right)\right]^{\frac{1}{2}}
$$

where $v_{g}$ is the group velocity, $\eta_{i}$ is the injection efficiency, $a$ is the differential gain, $q$ is electron charge, $V_{m}$ is the mode volume, $I$ is the bias current, and $I_{\text {th }}$ is the threshold current. 


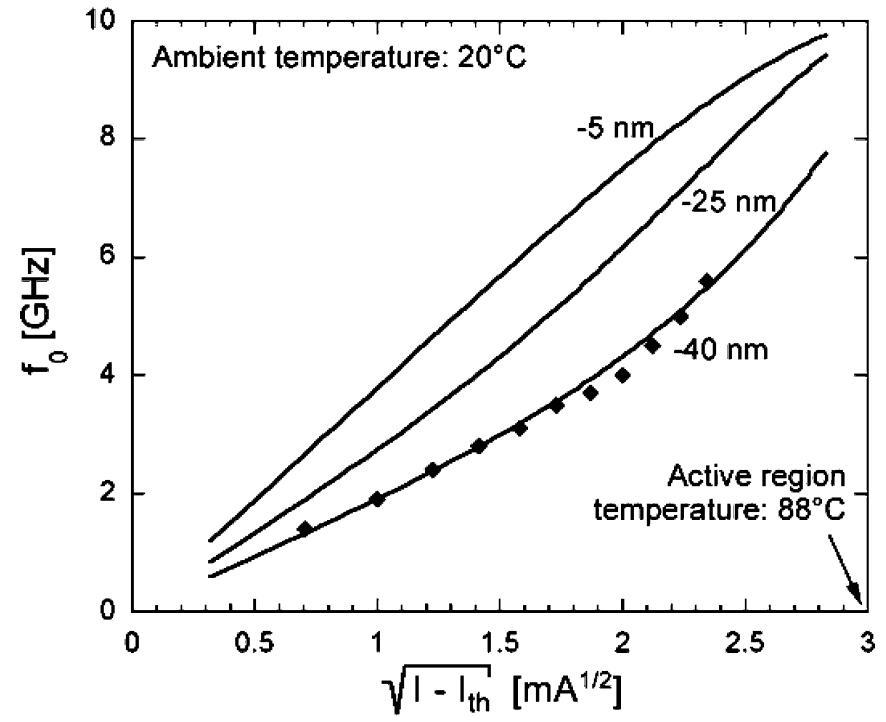

Fig. 4. Relaxation resonance frequency versus square root of the bias current less the threshold current. The dots show measured data; the lines were calculated from the theoretical gain curves shown in Fig. 2.

The active region temperature is related to the drive current by the expression $T=R_{\mathrm{th}}\left(I V-P_{\mathrm{out}}\right)$, where $R_{\mathrm{th}}$ is the thermal impedance (measured to be $1.3^{\circ} \mathrm{C} / \mathrm{mW}$ ), $V$ is the bias voltage, and $P_{\text {out }}$ is the VCSEL output power. The range of drive currents shown in Fig. 4 goes from threshold $(2 \mathrm{~mA})$ to $10 \mathrm{~mA}$, at which the internal temperature is estimated to be about $88^{\circ} \mathrm{C}$. Excellent agreement between the data and the calculations are demonstrated using $\eta_{i}=0.65$. The mode volume of the VCSEL used in this experiment was calculated to be $1.66 \times 10^{-12} \mathrm{~cm}^{3}$, and includes the field penetration depth into the distributed Bragg reflectors. From the measured data for -40-nm RT gain-mode offset, a modulation current efficiency of about $2 \mathrm{GHz} / \mathrm{mA}^{1 / 2}$ can be extracted at low drive currents. For higher drive currents, as the internal temperature increases due to self heating, the differential gain increases because of the change in gain-mode offset resulting in a local modulation current efficiency of $4.8 \mathrm{GHz} / \mathrm{mA}^{1 / 2}$.

As shown in Fig. 4, the highest resonance frequency at RT ambient is predicted for the smallest offset of $-5 \mathrm{~nm}$. However, with this offset, $f_{0}$ starts to roll off at higher drive currents due to the increase in active region temperature. At elevated temperatures, this offset results in further reduced differential gain (Fig. 2). This design is also poorly suited for high-temperature operation (Fig. 3). The -40 -nm offset used in the present devices is too large for good high-frequency modulation at low temperatures. At internal temperatures above $140{ }^{\circ} \mathrm{C}$, this offset produces the highest differential gain (Fig. 2). -25-nm gainmode offset results in the smallest differential gain variation across the temperature range shown in Fig. 2. At low temperatures, it shows higher differential gain than the design using -40-nm offset. Between $100{ }^{\circ} \mathrm{C}$ and $140{ }^{\circ} \mathrm{C},-25$-nm offset yields higher differential gain, and hence, higher $f_{0}$, than the other two offsets considered here.
In conclusion, the differential gain in long-wavelength $\mathrm{VC}$ SELs varies with gain-mode offset leading to variations in $f_{0}$ with internal temperature changes in the VCSEL. There is a design tradeoff between high-temperature operation and good modulation properties. The large negative gain-mode offsets commonly used to achieve operation at high temperatures result in compromised $f_{0}$. A small negative offset, or zero offset, would result in good modulation properties at low temperatures, but poor high-temperature performance. For the QW design analyzed here, an RT gain-mode offset of $-25 \mathrm{~nm}$ was shown to minimize the variation in differential gain over an internal temperature range of $20{ }^{\circ} \mathrm{C}$ to $140{ }^{\circ} \mathrm{C}$, and simultaneously produce satisfactory high-temperature operation. In order to further optimize the modulation properties of VCSELs, further reduction of the threshold current and the size of the optical mode must be addressed.

\section{REFERENCES}

[1] I. Suemune, L. A. Coldren, M. Yamanishi, and Y. Kan, "Extremely wide modulation bandwidth in a low threshold current strained quantum well laser," Appl. Phys. Lett., vol. 53, no. 15, pp. 1378-1380, Oct. 1988.

[2] K. Uomi, T. Mishima, and N. Chinone, "Ultrahigh relaxation oscillation frequency (up to $30 \mathrm{GHz}$ ) of highly p-doped GaAs/GaAlAs multiple quantum well lasers," Appl. Phys. Lett., vol. 51, no. 2, pp. 78-80, Jul. 1987.

[3] H. Nishimoto, M. Yamaguchi, I. Mito, and K. Kobayashi, "High-frequency response for DFB LD due to a wavelength detuning effect," $J$. Lightw. Technol., vol. LT-5, no. 10, pp. 1399-1402, Oct. 1987.

[4] S. Robert, M. Ortsiefer, J. Rosskopf, G. Böhm, C. Lauer, M. Maute, and M.-C. Amann, "Long-wavelength InP-based VCSELs with buried tunnel-junction: Properties and applications," Proc. SPIE, vol. 5364, pp. $1-15$, Jun. 2004.

[5] S. Nakagawa, E. Hall, G. Almuneau, J. K. Kim, D. A. Buell, H. Kroemer, and L. A. Coldren, " $88^{\circ} \mathrm{C}$ continuous wave operation of apertured intracavity contacted $1.55-\mu \mathrm{m}$ vertical-cavity surface emitting lasers," Appl. Phys. Lett., vol. 78, no. 10, pp. 1337-1339, Mar. 2001.

[6] R. Shau, M. Ortsiefer, J. Rosskopf, G. Bohm, F. Kohler, and M. C. Amann, "Vertical cavity surface-emitting laser diodes at $1.55 \mu \mathrm{m}$ with large output power and high operation temperature," Electron. Lett., vol. 37, no. 21, pp. 1295-1296, Oct. 2001.

[7] V. Jayaraman, M. Mehta, A. W. Jackson, S. Wu, Y. Okuno, J. Piprek, and J. E. Bowers, "High-power 1320-nm wafer-bonded VCSELs with tunnel junctions," IEEE Photon. Technol. Lett., vol. 15, no. 11, pp. 1495-1497, Nov. 2003.

[8] A. Syrbu, A. Mircea, A. Caliman, C.-A. Berseth, G. Suruceanu, V. Iakovlev, M. Achtenhagen, A. Rudra, and E. Kapon, "1.5-mW single-mode operation of wafer-fused 1550-nm VCSELs," IEEE Photon. Technol. Lett., vol. 16, no. 5, pp. 1230-1232, May 2004.

[9] P. A. Morton, T. Tanbun-Ek, R. A. Logan, P. F. Sciortino Jun, A. M. Sergent, and K. W. Wecht, "Superfast $1.55 \mu \mathrm{m}$ DFB lasers," Electron. Lett., vol. 29, no. 16, pp. 1429-1430, Aug. 1993.

[10] H. D. Summers, P. Dowd, I. H. White, and M. R. T. Tan, "Calculation of differential gain and linewidth enhancement factor in 980-nm InGaAs vertical cavity surface-emitting lasers," IEEE Photon. Technol. Lett., vol. 7, no. 7, pp. 736-738, Jul. 1995.

[11] P. Dowd, H. D. Summers, I. H. White, M. R. T. Tan, Y. M. Houng, and S. Y. Wang, "Measurement of differential gain and linewidth enhancement factor of InGaAs vertical cavity surface emitting laser," Electron. Lett., vol. 31, no. 7, pp. 557-559, Mar. 1995.

[12] J. Piprek, M. Mehta, and V. Jayaraman, "Design and optimization of high-performance $1.3 \mu \mathrm{m}$ VCSELs," in Proc. SPIE, Physics and Simulation of Optoelectronic Devices XII, Photonics West 2004, vol. 5349-49, 2004, pp. 375-384.

[13] L. A. Coldren and S. W. Corzine, Diode Lasers and Photonic Integrated Circuits. New York: Wiley, 1995. 